\title{
Altering the pattern of panretinal photocoagulation: could the visual field for driving be preserved?

\begin{abstract}
Purpose To identify the area of retina required to provide the visual field for driving and to investigate whether the pattern of panretinal photocoagulation (PRP) for proliferative diabetic retinopathy could be altered to avoid treatment in this area whilst leaving the total number of burns constant.

Methods A mathematical model of the emmetropic eye is used to calculate retinal dimensions corresponding to different angles of visual field. These are used to define retinal regions that correspond to the UK DVLC visual field criteria and regions that lie outside this area. Further calculation estimates the number of laser burns applied within these regions for both $500 \mu \mathrm{m}$ and $200 \mu \mathrm{m}$ diameter spot sizes and various burn spacings.

Results Modelling of the number of burns applied in the normal pattern of PRP agrees with the number required to control proliferative retinopathy. Reducing burn spacing or extending treatment up to the ora serrata allows application of sufficient burns to control the disease without encroaching on areas of the retina that provide the driving field.

Conclusion It is theoretically possible to alter the pattern of PRP to avoid treatment in retinal areas concerned with the driving visual field whilst leaving the total number of burns constant. This suggests that a clinical trial of such a pattern PRP could be performed to assess adequate control of proliferative retinopathy along with preservation of the visual field required for driving.
\end{abstract}

Key words Diabetes, Driving, Panretinal photocoagulation, Proliferative retinopathy

Panretinal photocoagulation (PRP) is the initial treatment of choice in proliferative diabetic retinopathy. The Diabetic Retinopathy Study (DRS) showed up to a $50 \%$ reduction of risk of severe visual loss. ${ }^{1}$ PRP itself has a well-known list of complications amongst which is the risk of constriction of peripheral visual field. ${ }^{1-5}$ The UK DVLC regulations require a visual acuity equal to or better than 3.125 inches at 67 feet and a binocular visual field of $120^{\circ}$ in the horizontal meridian and no significant scotoma within $20^{\circ}$ of fixation above and below the horizontal midline. ${ }^{6}$ There have been several papers recently concerning the loss of visual field after PRP and the effect on the patients' legal status for driving. ${ }^{7-10}$

In these studies, the percentage of patients failing the visual field test varied from $12 \%{ }^{10}$ to $50 \%, 7$ although the latter included 5 patients who had had xenon arc photocoagulation (4/5 failed). Considering only those who had argon laser treatment the failure rate was $44 \%$. Only one of these studies was prospective in its design $^{10}$ and this showed a failure rate of $12 \%$ with bilateral PRP and a $500 \mu \mathrm{m}$ spot size. It has been suggested that using a smaller laser spot size of $200 \mu \mathrm{m}$ diameter might be associated with a higher pass rate, ${ }^{8}$ although this conclusion was based on retrospective analysis of patients undergoing PRP, many of whom had had different spot sizes applied to the same eye. The authors of this study rightly state that a prospective randomised trial would give a clearer answer to this question and they estimated a total of 600 patients would be needed to obtain a meaningful result.

The usual pattern for PRP is to apply burns in a scatter fashion around the retina, avoiding the posterior pole and reaching up to the level of the vortex veins (equator). The burn spacing is one half burn diameter for $500 \mu \mathrm{m}$ burns and one burn diameter for $200 \mu \mathrm{m}$ burns. The DRS initially recommended application of 800-1600 $500 \mu \mathrm{m}$ burns, but this was later amended to $1200-1600$ burns as the lower figure was found to be inadequate. ${ }^{11}$ Hulbert and Vernon ${ }^{8}$ suggest that 3000-3500 $200 \mu \mathrm{m}$ burns are sufficient to control the disease in all but severe cases and Mackie et al. ${ }^{9}$ state that at least 3000 $200 \mu \mathrm{m}$ burns should be applied.

PRP results in laser burns within the area of retina that provides the individual with the visual field deemed necessary for driving. The
N. Davies

Vision Group

Physics (Biophysics)

Blackett Laboratory Imperial College Science, Technology and Medicine Prince Consort Road South Kensington London SW7 2BZ, UK

Tel: +44 (0)171 594 7674/2 e-mail: n.p.davies@ic.ac.uk

Received: 22 September 1998

Accepted in revised form: 8 March 1999 


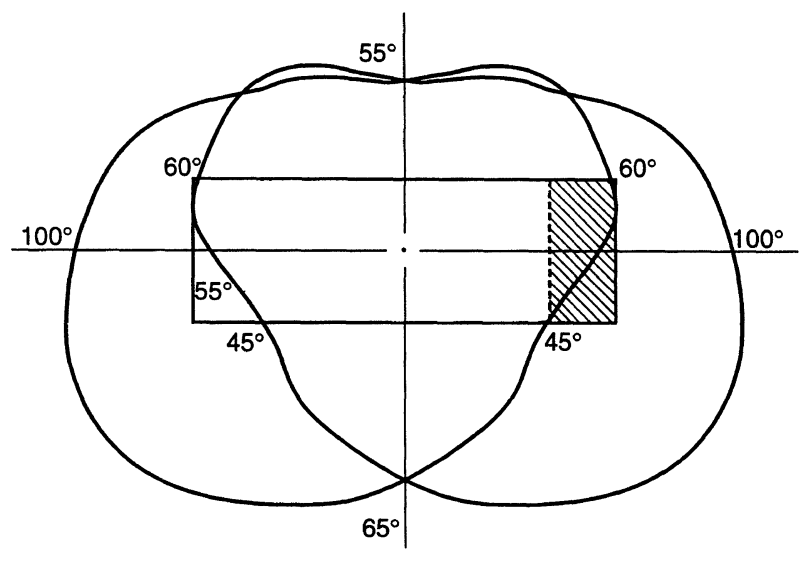

Fig. 1. Binocular visual field. The central rectangle represents the visual field requirements of the DVLC. The hatched area represents a 'safe' monocular crescent: see text.

intriguing question arises whether these areas of the retina could remain unablated after PRP in order to preserve as much of the driving visual field (DVF) as possible. This raises two further questions: Is there sufficient retina available elsewhere to accommodate the required number of burns, and will altering the burn pattern, whilst maintaining the total number of burns constant, still allow adequate control of the disease? This paper addresses the first of these questions using a mathematical model.

\section{Method}

The model used assumes a spherical emmetropic globe. It also assumes laser burns applied are circular and not magnified by the fundus lens. These assumptions simplify the mathematics and allow an estimation of the number of burns applied to different parts of the retina to a reasonable degree of accuracy.

The retina is divided into a series of regions, each of which provides a defined area of visual field. This is followed by calculation of the number of burns applied in each region for different burn sizes and spacing. To avoid excessive recourse to mathematics, which can be tedious to follow, the results will be quoted directly after definition of the variables involved. The full derivations of the formulae used are given in the Appendix.

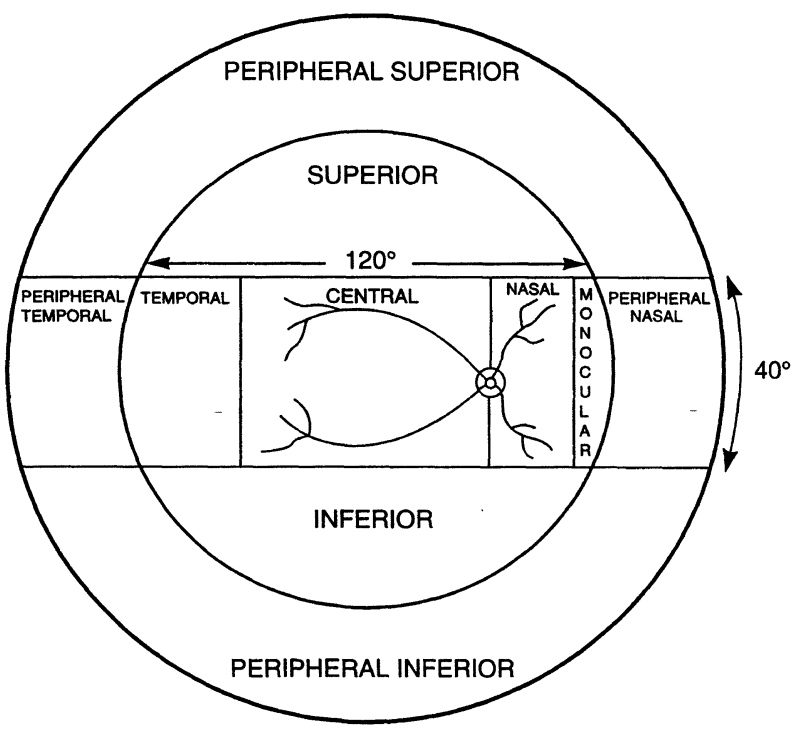

Fig. 2. Schematic of definitions of retinal regions. Outer circle represents the ora serrata, inner circle the equator.

The calculations are based on a consideration of a binocular patient requiring either unilateral or bilateral PRP. It is important to consider the relationship between the visual fields of each eye as this has implications in any attempt to preserve the DVF. The more temporal aspect of each field is monocular, forming the temporal monocular crescent. At the horizontal midline the binocular field extends to around $55^{\circ}$ from fixation in each direction. At $20^{\circ}$ above the midline it extends to $60^{\circ}$ from fixation and $20^{\circ}$ below the midline it extends to $45^{\circ}$ from fixation. The binocular visual field and the most stringent interpretation of the field required for driving are shown in Fig. 1.

In the case of a binocular patient requiring unilateral PRP only the temporal monocular crescent lying within the driving field needs to be left untreated to allow the DVF to remain intact. To ensure this is the case it would be sensible to exclude a rectangular area extending from $45^{\circ}$ to just beyond $60^{\circ}$ horizontally, reaching to $20^{\circ}$ both above and below the horizontal midline. This is shown as a hatched area in Fig. 1.

Table 1. Definition of retinal regions

\begin{tabular}{|c|c|}
\hline \multicolumn{2}{|l|}{ Central regions } \\
\hline Nasal region & Nasal margin of disc up to the equator, vertical width $40^{\circ}$ \\
\hline Monocular region & Lateral edge of the nasal region up to the equator, vertical width \\
\hline Temporal region & One disc-fovea distance temporal to the fovea up to the equator, \\
\hline Central region & Area between the nasal and temporal regions, with the same ver \\
\hline Inferior region & Inferior border of the above four regions up to the equator inferi \\
\hline Superior region & Superiorly from the above four regions up to the equator superic \\
\hline \multicolumn{2}{|l|}{ Peripheral regions } \\
\hline Peripheral nasal & Nasal aspect of the equator up to the ora, vertical width $40^{\circ}$ \\
\hline Peripheral temporal & Temporal aspect of the equator up to the ora, vertical width $40^{\circ}$ \\
\hline Peripheral superior & Superior border of the superior region up to the ora \\
\hline Peripheral inferior & Inferior border of the inferior region up to the ora \\
\hline
\end{tabular}


In bilateral PRP one eye can be treated as in the unilateral case, but in the second eye treatment needs to be avoided in both the temporal and nasal areas of the retina that provide the DVF.

\section{Definition of retinal regions}

The dimensions of the retinal regions are chosen such that each provides a defined area of visual field.

Equation (3) from the Appendix shows that the equator of an emmetropic eye projects to $61.5^{\circ}$ of visual field. This makes definition and calculation of the corresponding retinal areas straightforward as the equator conveniently projects to just beyond $60^{\circ}$ from fixation. Let the retina be divided into two main portions: central and peripheral. The central regions lie posterior to the equator, peripheral regions anterior to the equator. These regions can be further subdivided into areas defined by the visual criteria for driving (Table 1, Fig. 2).

\section{Results}

Initially it is necessary to calculate the arc lengths of each retinal region. Let each arc length be $b_{\psi} \mathrm{mm}$ where $\psi$ is the degrees of visual field provided by that length of retina. The vertical width of the central regions is each equivalent to $40^{\circ}$ of visual field, so $b_{40}$ needs to be found. Also $b_{15}$ is needed to know the disc-fovea distance and $b_{46.5}$ is used to find the limit of the nasal region. The anterior limit of the regions has been defined as the equator (which projects to $61.5^{\circ}$ of visual field). The value of $b_{61.5}$ can readily be calculated. It is also necessary to find $b_{\text {ora }}$ as this gives the retinal dimension from fixation to the ora serrata. These dimensions are found using formulae (1), (2) and (3) from the Appendix; the results are presented in Table 2.

\section{Calculation of the number of burns applied during PRP in each retinal region}

The number of burns in each retinal region can be calculated using equations (4) to (7) from the Appendix for any given burn diameter and spacing.

Tables 3 and 4 give the total number of burns in the different retinal regions with the different specified diameters and spacing. Note that the number of $500 \mu \mathrm{m}$ burns spaced $250 \mu \mathrm{m}$ apart and $200 \mu \mathrm{m}$ burns spaced $200 \mu \mathrm{m}$ apart applied up to the equator agree with the number recommended to control the disease.

Table 2. Retinal arc length with respect to angle of visual field

\begin{tabular}{lc}
\hline Field $(\psi)$ & Retinal arc length $\left(b_{\psi}\right) \mathrm{mm}$ \\
\hline $15^{\circ}$ & 4.45 \\
$40^{\circ}$ & 11.65 \\
$46.5^{\circ}$ & 13.03 \\
Equator $\left(61.5^{\circ}\right)$ & 17.37 \\
Ora serrata & 21.70 \\
\hline
\end{tabular}

Table 3. Number of burns in each retinal region $(500 \mu \mathrm{m})$

\begin{tabular}{lccc}
\hline & \multicolumn{3}{c}{ Spacing between burns } \\
\cline { 2 - 4 } Region & $500 \mu \mathrm{m}$ & $250 \mu \mathrm{m}$ & $125 \mu \mathrm{m}$ \\
\hline Monocular & 50 & 90 & 129 \\
Nasal & 100 & 177 & 256 \\
Temporal & 150 & 267 & 385 \\
Inferior & 225 & 403 & 575 \\
Superior & 225 & 403 & 575 \\
Total to equator & 750 & 1340 & 1900 \\
Peripheral nasal & 50 & 89 & 129 \\
Peripheral temporal & 50 & 89 & 129 \\
Peripheral inferior & 90 & 179 & 251 \\
Peripheral superior & 90 & 179 & 251 \\
Total to ora & 1030 & 1876 & 2660 \\
\hline
\end{tabular}

\section{Altering the burn pattern}

To ascertain whether the pattern of PRP could be altered to avoid treatment in the retinal region, providing a DVF whilst keeping the total number of burns constant, the data presented in Tables 3 and 4 need to be studied further. For example, with a $200 \mu \mathrm{m}$ spot size, decreasing the burn spacing from $200 \mu \mathrm{m}$ to $100 \mu \mathrm{m}$ in the inferior region allows placement of an extra 1087 burns (2404-1317). The results of the calculations like the above are presented below.

\section{$200 \mu m$ burns}

1. In unilateral PRP the monocular region is left untreated. This would entail repositioning only 316 burns and the most obvious site would be the peripheral nasal region as this can accept the same number.

2. If or when PRP is required in the second eye a more radical change is needed in order to try to preserve the driving field. This would require that the monocular, nasal and temporal regions of the second eye remain untreated. This entails repositioning a total of 1882 burns and could be done in different ways:

(a) Altering the burn spacing to one half a burn diameter in both the superior and inferior regions would allow the placement of a potential extra 2174 burns, which

Table 4. Number of burns in each retinal region (200 $\mu \mathrm{m})$

\begin{tabular}{lcc}
\hline & \multicolumn{2}{c}{ Spacing between burns } \\
\cline { 2 - 3 } Region & $200 \mu \mathrm{m}$ & $100 \mu \mathrm{m}$ \\
\hline Monocular & 316 & 561 \\
Nasal & 625 & 1111 \\
Temporal & 941 & 1673 \\
Inferior & 1317 & 2404 \\
Superior & 1317 & 2404 \\
Total to equator & 4517 & 8154 \\
Peripheral nasal & 315 & 560 \\
Peripheral temporal & 315 & 560 \\
Peripheral inferior & 620 & 1051 \\
Peripheral superior & 620 & 1051 \\
Total to ora & 6387 & 11376 \\
\hline
\end{tabular}


easily accepts the burns from the untreated regions. This would give a burn pattern of high density superiorly and inferiorly.

(b) Burn spacing could be reduced to $100 \mu \mathrm{m}$ inferiorly, accommodating an extra 1087 burns, and the treatment continued to include the peripheral inferior and either the peripheral nasal or peripheral temporal regions (a further 935 burns), which again accepts the required number.

(c) The spacing between burns could be reduced to 100 $\mu \mathrm{m}$ in both the inferior and peripheral inferior regions. This allows an extra 2138 burns to be placed.

(d) The burn spacing could be left at $200 \mu \mathrm{m}$ and all the peripheral regions treated. A total of 1870 extra burns could be applied.

\section{$500 \mu m$ burns}

In the case of the larger burn size the inter-burn spacing is already at $250 \mu \mathrm{m}$ in order to apply the requisite number of around 1200-1600 burns.

1. In unilateral PRP the monocular region should be left untreated, which would require placement of 90 burns elsewhere. These can be accommodated in the peripheral nasal region.

2. To avoid the driving field in patients undergoing PRP in their second eye, the monocular, nasal and temporal regions should be left untreated. This requires repositioning of 534 burns. This again can be accomplished in different ways:

(a) In the inferior and superior regions the spacing can be reduced to $125 \mu \mathrm{m}$. This would accommodate an extra 586 burns.

(b) The inferior, peripheral inferior and either the peripheral nasal or peripheral temporal regions can be treated at a spacing of $125 \mu \mathrm{m}$. This would accommodate an extra 552 burns.

(c) If all the peripheral regions are treated at a spacing of $250 \mu \mathrm{m}$ then a total of 536 extra burns could be placed.

Altering the pattern of $500 \mu \mathrm{m}$ burns to avoid the driving field is feasible. However, the burns have to be tightly packed elsewhere (one quarter of a diameter) unless the whole fundus up to the ora is treated.

\section{Discussion}

A series of equations have been derived that can be used to calculate arc lengths of defined retinal regions and the number of laser burns that can be applied within them. The data show that it is theoretically possible to alter the pattern of PRP in an attempt to preserve a visual field that conforms to the DVLC requirements, bearing in mind that some diabetic patients have visual field defects which pre-date any iatrogenic loss. ${ }^{12-14}$ It is easier to avoid the DVF with a $200 \mu \mathrm{m}$ burn than with a $500 \mu \mathrm{m}$ burn. This is probably because $500 \mu \mathrm{m}$ burns have to be spaced $250 \mu \mathrm{m}$ apart initially and suitable alteration of the pattern produces quite small inter-burn spacing unless the whole fundus is treated. The analysis does not intend to present an exhaustive list of combinations of different burn patterns possible to preserve driving visual field but rather indicates its feasibility.

It is not possible from the above to gain any knowledge of how changing the pattern of PRP, even whilst keeping the total number of burns constant, would affect control of proliferative diabetic retinopathy. Blankenship ${ }^{11}$ conducted a trial comparing 'peripheral PRP' with 'central PRP', where a 'peripheral PRP' involved treatment of mid-peripheral fundus with a panfundoscope lens and then up to the ora with a Goldmann three-mirror lens using $500 \mu \mathrm{m}$ burns. 'Central PRP' used the same pattern with the panfundoscope but the Goldmann lens was used to treat the posterior fundus, avoiding an area 2 disc diameters wide, centred on the fovea. This trial showed that each form of PRP produced adequate regression of the new vessels in the majority of cases. There was no significant difference between the two groups in the number of patients who required further treatment. More patients undergoing central PRP lost 2 or more lines of visual acuity than those with peripheral treatment, although the difference was not significant. Visual field testing showed that with both the IVe and I4e targets, a greater loss of field occurred in those patients having central rather than peripheral treatment. However, the sample size was quite small and a larger number of patients would be needed to look for a statistically significant difference in visual acuity and in field loss. This study is encouraging in that it showed adequate control of neovascularisation with two differing patterns of treatment.

In order to maximise the probability of controlling proliferative retinopathy with a new distribution of burns, the choice of pattern is likely to be important. Although the peripheral PRP mentioned above did result in adequate new vessel regression, it would seem sensible to apply as many laser burns in the postequatorial region as possible. For example the pattern (2a) in the results section for either $200 \mu \mathrm{m}$ or $500 \mu \mathrm{m}$ spot diameters gives the required number of burns overall by reducing burn spacing in the superior and inferior regions without lasering into the periphery. This may be advantageous in application of the treatment and also in controlling the proliferation of new vessels.

In the clinical implementation of such a pattern PRP, the model could be tailored to suit each patient's globe radius to allow calculation of the exact retinal dimensions corresponding to the driving visual field. This would control for the inter-individual variation in globe size and allow accurate placement of laser burns to avoid the driving field.

A randomised controlled clinical trial would therefore seem appropriate, comparing the visual fields of patients undergoing a pattern treatment aimed at preserving driving field with patients undergoing a scatter PRP. If the altered pattern PRP gives control of proliferative retinopathy and preserves field the new pattern could be adopted. However, if the retinopathy were not controlled 
to an acceptable degree, the standard scatter PRP should remain the treatment of choice and ophthalmologists would be in a position to warn patients that potential loss of their driving field is an unavoidable risk of treatment for the benefit of maintaining their central vision.

\section{Appendix}

Calculation of arc lengths of retina with respect to angles of visual field

To calculate the retinal dimensions of the regions subserving the different areas of visual field consider Fig. 3:

Arc length subtended by angle $\psi$ of visual field $=b_{\psi}$ Internal radius of emmetropic globe $r=11.06$

(Taylor and Dobree ${ }^{14}$ )

Distance from nodal point to fovea $=x+y+z=17.05 \mathrm{~mm}$.

To calculate the arc length $b$ one needs to determine $\theta$ in terms of $\psi$

From Fig. 3: $w=y \sin \psi=r \sin \theta$

$$
\text { therefore } \quad \begin{aligned}
x+y=v & \cos \psi \quad \text { and } y=r \cos \theta \\
x+r \cos \theta & =\sin \theta \cdot \cos \psi / \sin \psi \\
& =r \sin \theta / \tan \psi
\end{aligned}
$$

If we now let $c=x / r$ and $k=1 / \tan \psi$, then

$$
\mathbf{c}+\cos \theta=\mathbf{k} \sin \theta
$$

Generally, $\sin ^{2} \theta+\cos ^{2} \theta=1$ and therefore $\cos \theta=$ $\left(1-\sin ^{2} \theta\right)^{1 / 2}$

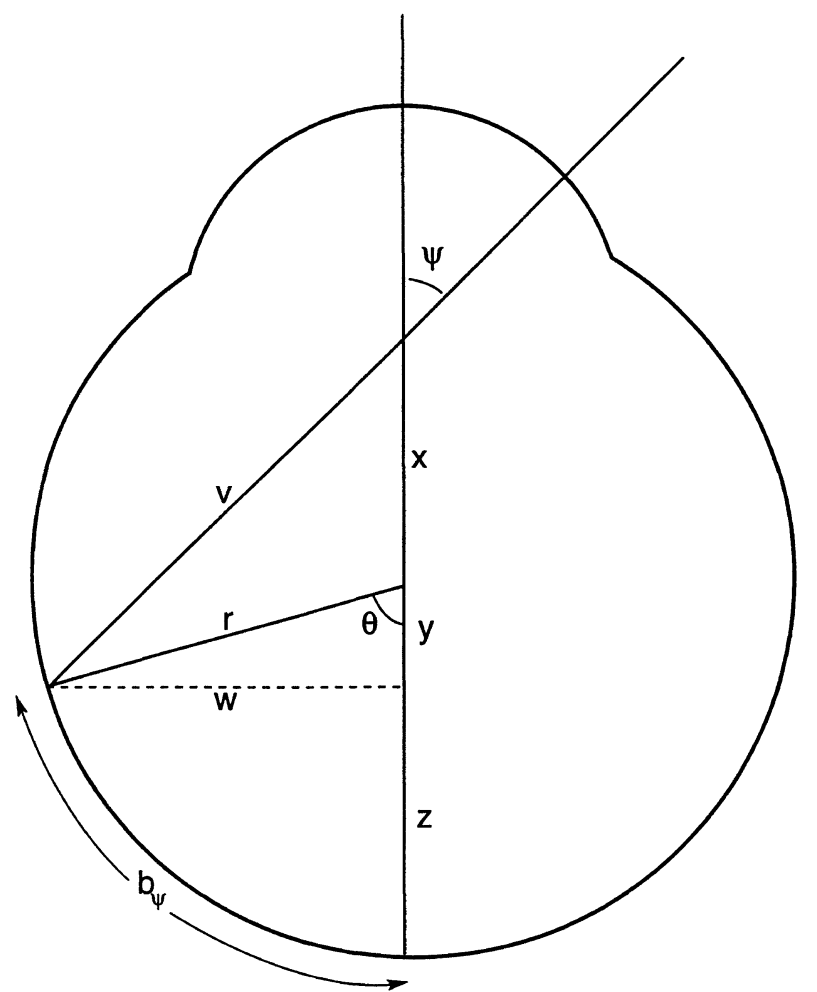

Fig. 3. Calculation of retinal dimension from given angle of visual field. A function describing $\theta$ in terms of $\psi$ is required to allow the calculation of $\mathrm{b}_{\psi}$.

$$
\left(1-\sin ^{2} \theta\right)^{1 / 2}=k \sin \theta-c
$$

and thus

$$
\left(k^{2}+1\right) \sin ^{2} \theta-2 k c \sin \theta+\left(c^{2}-1\right)=0
$$

Solving the quadratic equation for $\sin \theta$ and taking the positive value gives

$$
\sin \theta=\left\{\mathbf{k} \mathbf{c}+\left(\mathbf{k}^{2}-\mathbf{c}^{2}+1^{1 / 2}\right\} /\left\{\mathbf{k}^{2}+1\right\}\right.
$$

$k$ and $c$ can be calculated from the known values of $r, x$ and $\psi$. Thus values for $\theta$ and hence $b$ can be calculated for different angles of $\psi$ from the equation for arc length of a circle:

$$
\mathbf{b}_{\psi}=\mathbf{r} \theta
$$

\section{Calculation of the number of burns over a given area of the retina}

Here an assumption is made that the burns are circular in shape and have a diameter $d$. It is also assumed that they are applied with a non-magnifying fundus lens throughout, which gives a burn size approximately equal to the laser spot size. The burn spacing is defined as $d^{\prime \prime}$.

\section{Rectangular retinal region (Fig. 4)}

Consider a row of $n$ burns across a length of retina $b$ :

Retinal length $b=n \cdot$ burn diameter $+n \cdot$ burn spacing Let the spacing between rows (i.e. column spacing) be $d^{\prime \prime}$. This gives that

$$
b_{\psi 1}=n\left(d+d^{\prime}\right)
$$

and

$$
b_{\psi 2}=n^{\prime}\left(d+d^{\prime \prime}\right)
$$

and the total number of burns over the rectangular area is thus:

$$
\begin{gathered}
N=n \cdot n^{\prime} \\
\mathbf{N}=\mathbf{b}_{\psi 1} \cdot \mathbf{b}_{\psi 2} /\left(\mathbf{d}+\mathbf{d}^{\prime}\right)\left(\mathbf{d}+\mathbf{d}^{\prime \prime}\right)
\end{gathered}
$$

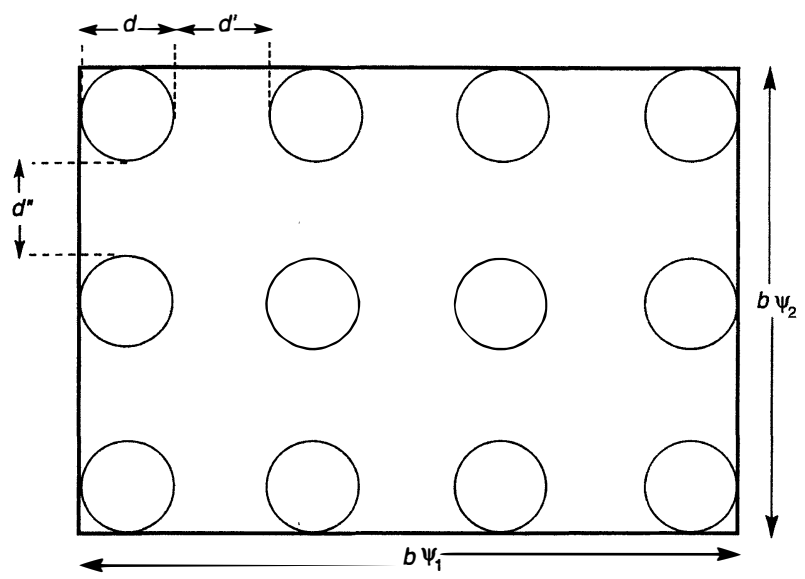

Fig. 4. Number of circular burns of diameter $\mathrm{d}$, spacing $\mathrm{d}$ ' that can be applied to a rectangular area of retina of dimensions $\mathrm{b}_{\psi_{1}}$ and $\mathrm{b}_{\psi_{2}}$. 


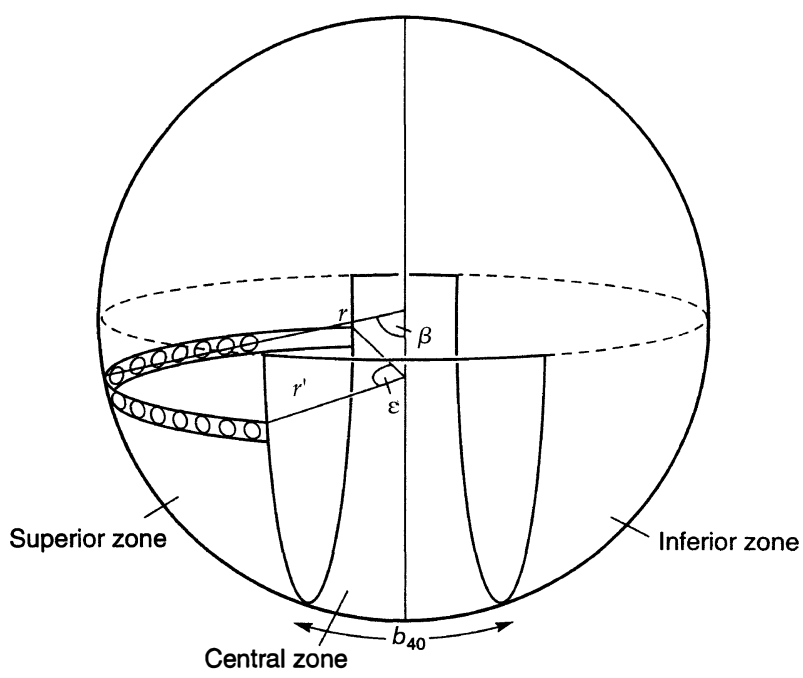

Fig. 5. Number of circular burns of diameter $\mathrm{d}$, spacing $\mathrm{d}$ ' than can be applied in a row to superior or inferior retinal regions at angle $\beta$ to the fovea. The total number of burns in each region is obtained by summing the number of burns applied in each row over the total number of rows.

Equation (4) can be used to calculate the number of burns applied in a rectangular area of retina.

For the superior and inferior regions the same general principle applies, although one needs to take into account the varying length of the rows of burns from the margins of the central regions up to the equator.

Consider Fig. 5.

The length of the row of burns shown is $r^{\prime} \varepsilon$, where $\varepsilon$ is the arc angle subtended.

So

$$
\varepsilon=\pi-b_{40} / r
$$

but we also know that

$r^{\prime}=r \sin \theta$

therefore

$$
\varepsilon r^{\prime}=r\left(\pi-b_{40} / r\right) \sin \beta
$$

and so the total number of burns per row, $n$, is

$$
n \cdot d+n \cdot d^{\prime}=r\left(\pi-b_{40} / r\right) \sin \beta
$$

so we have the general result

$$
n=r\left(\pi-b_{40} / r\right) \sin \beta /\left(d+d^{\prime}\right)
$$

The total number of rows of burns $\left(n^{\prime}\right)$ is found from a consideration of the angle $\beta$. Let the first row (row zero) be closest to the border of the central regions. Each subsequent row is then at a larger angle of $\beta$, which is defined by the burn spacing between rows $\left(d^{\prime \prime}\right)$. i.e.

$$
\beta_{0}=b_{40} / 2 r
$$

so

$$
\beta_{1}=b_{40} / 2 r+\left(d+d^{\prime \prime}\right) / r
$$

and

$$
\beta_{\mathrm{n}}{ }^{\prime}=\mathrm{b}_{40} / 2 \mathrm{r}+\mathrm{n}^{\prime}\left(d+d^{\prime \prime}\right) / r
$$

The upper limit of $\beta$ is the equator by definition (i.e. $\pi / 2$ ):

therefore

$$
\pi / 2=b_{40} / 2 r+\mathrm{n}^{\prime}\left(d+d^{\prime \prime}\right) / r
$$

$$
\mathbf{n}^{\prime}=\mathbf{r}\left(\pi / 2-\mathbf{b}_{40} / \mathbf{r}\right) /\left(\mathbf{d}+\mathbf{d}^{\prime \prime}\right)
$$

The overall number of burns in the regions is the sum of $n$ over the range of values of $\beta$. This is:

$$
\mathbf{N}=\mathbf{r}\left(\pi-\mathbf{b}_{40} / \mathbf{r}\right) /\left(\mathbf{d}+\mathbf{d}^{\prime}\right) \cdot \Sigma_{\beta} \sin \beta
$$

where

$$
\beta=b_{40} / 2 r \text { to } \pi / 2 \text { in steps of }\left(d+d^{\prime \prime}\right) / r
$$

This last series of equations can be used to calculate the number of burns applied with different inter-burn spacings in the inferior and superior regions. Equation (5) will give the number of burns in a given row.

Equation (6) will give the total number of rows. Finally, equation (7) gives the number of burns over the retinal region, by summing the number of burns per row over the number of rows.

\section{References}

1. The Diabetic Retinopathy Research Group. Photocoagulation treatment of proliferative diabetic retinopathy: the second report of diabetic retinopathy study findings. Ophthalmology 1978;85:82-106.

2. Frank RN. Visual fields and electroretinography following extensive photocoagulation. Arch Ophthalmol 1975;93:591-8.

3. Rogell GD. Incremental panretinal photocoagulation. Retina 1983;3:308-11.

4. Russell PW, Sekuler R, Fetkerhur C. Visual function after panretinal photocoagulation. Diabetes Care 1985;8:57-63.

5. Hamilton AM, Townsend C, Khoury D, Gould E, Blach RK. Xenon arc and argon laser photocoagulation in the treatment of diabetic disc neovascularisation. I. Effect on disc vessels, visual fields and visual acuity. Trans Ophthalmol Soc UK 1981;101:87-92.

6. Form CLE 1060. DVLC drivers medical branch, revised 1992.

7. Buckley SA, Jenkins L, Benjamin L. Fields, DVLC and panretinal photocoagulation. Eye 1992;6:623-6.

8. Hulbert MFG, Vernon SA. Passing the DVLC field regulations following bilateral panretinal photocoagulation in diabetics. Eye 1992;6:456-61.

9. Mackie SW, Webb LA, Hutchinson BM, Hammer HM, Barrie T, Walsh G. How much blame can be placed on laser photocoagulation for failure to attain driving standards? Eye 1995;9:517-25.

10. Pearson AR, Tanner V, Keightly SJ, Casswell AG. What effect does laser photocoagulation have on driving visual fields in diabetics? Eye 1998;12:64-8.

11. Blankenship GW. A clinical comparison of central and peripheral argon laser panretinal photocoagulation for proliferative diabetic retinopathy. Ophthalmology 1988;95:170-7.

12. Trick GL, Trick LR, Kilo C. Visual field defects in patients with insulin-dependent and non-insulin-dependent diabetes. Ophthalmology 1990;97:475-82.

13. Wisznia KI, Lieberman TW, Leopold IH. Visual fields in diabetic retinopathy. Br J Ophthalmol 1971;55:183-8.

14. Taylor E, Dobree.JH. Proliferative diabetic retinopathy: site and size of initial lesions. Br J Ophthalmol 1970;54:11-8.

15. Taylor E, Jennings A. Calculation of total retinal area. $\mathrm{Br} \mathrm{J}$ Ophthalmol 1971;55:262-5. 